\title{
An Approach for obtaining Least Noisy Signal using Kaiser Window and Genetic Algorithm
}

\author{
Poulami Das \\ Research Scholar \\ Dept. of CSE \\ Jadavpur University
}

\author{
Subhas Chandra \\ Panja \\ Asst. Prof. \\ Dept. of Mechanical \\ Engineering \\ Jadavpur University
}

\author{
Sudip Kumar \\ Naskar \\ Asst. Prof. \\ Dept. of CSE \\ Jadavpur University
}

\author{
Sankar Narayan \\ Patra \\ Asst. Prof. \\ Dept. of \\ Instrumentation \\ Science \\ Jadavpur University
}

\begin{abstract}
During transmission via any media signals get affected by unwanted components; which is adverse but inevitable. Elimination of such unwanted components termed as noise from transmitted signals persisted important as well as puzzling task for the researchers from the initial days of Digital Signal Processing. Among a significant number of techniques proposed for removal of noise from signals, use of digital filters has become most effectual in multiple ways. Slighter overheads in designing and lower hardware cost have made the Finite Impulse Response (FIR) filters popular. FIR filter is expansively used in video convolution functions, signal preconditioning, and various communication applications. Till date, most of the FIR filter designing techniques is based on Window method, Optimal Sampling Method, Frequency Sampling Method. In this paper a new subterfuge based on Genetic Operators and Kaiser Window function has been proposed to obtain the least noisy signal from a set of filtered signals of a corrupted audio signal.
\end{abstract}

\section{General Terms}

Finite Impulse Response Filter, Window Function, Kaiser Window, Signal De-noising, Genetic Algorithm.

\section{Keywords}

Finite Impulse Response Filter, Roulette wheel selection technique, Kaiser Window, Genetic Algorithm, off spring, Signal to Noise Ratio (SNR), Beta Factor.

\section{INTRODUCTION}

Signal transmission habitually includes unwanted components (noise) in the signal. Exclusion of noise from transmitted signals still remains a research hotspot for the researchers in the field of signal processing. Since the earliest days of discrete time systems, both the types of FIR and IIR systems have gone through several advancements in different eras. A considerable number of algorithms [1-3] have been proposed by several researchers for designing digital filters over the years. For lesser overheads in designing and lower hardware cost, FIR filters has become popular than the IIR $[4,5]$ in past few epochs. FIR filter is extensively used in video convolution functions, signal preconditioning and various communication applications.

Design of digital FIR filters involves calculation of filter transfer function coefficients $h_{n}, n=0,1, \ldots, N$ that provide target frequency response. A number of methods like window method, optimal sampling and frequency sampling have been introduced for this purpose. A wide number of windowing methods $[7,8,9]$ were proposed earlier, based on different parameters like sampling frequency, cut off frequency, passband ripple, stopband attenuation, order of the filter, filter length, width of the lobes, etc. Most basic window function is rectangular window function where the signal is truncated by multiplying the impulse response with a function with unit amplitude within a given range and ignoring the coefficients outside the window. It was mentioned [10] the presence of overshoots and ripples in frequency response due to the high oscillation or side lobes caused by abrupt truncation. To reduce these effects, windows that do not contain abrupt discontinuities in their time and frequency domain characteristics are chosen. To overcome the limitations of rectangular window function, few windows were introduced for the implementation of FIR digital filters that might not contain hasty discontinuity in time and frequency domain characteristics. In this regard, most commonly used windows are Hamming [11], Hanning [12], Exponential window [13], Blackman [14], Chebyshev [14]. As these methods suffer from the lack of design flexibility, Kaiser Window has been used to obtain filtered signals in the research work.

In this work, Kaiser Window [15] with varying passband and stopband ripples has been used. Kaiser window function [16] was first proposed by Kaiser to design non-recursive digital filters by using the modified zeroth order Bessel function $\left(\mathrm{I}_{0^{-}}\right.$ sinh). Later, Kaiser Window $[17,18]$ has gone through several modifications proposed by different researchers. Kaiser window is detailed in Section-II of this paper. The efficacy of the proposed algorithm is tested on noisy audio signal in the present work. At the very first step, filtration is executed by several filters implemented using Kaiser Window function varying ripple factors. Thus a set of filtered signals is obtained. In the next step the approach behind the most commonly used evolutionary search algorithm namely concept of Genetic Algorithm [19, 20, 21, 22] is used to find out the optimum signal with lowest amount of noise by using the set of filtered signals as initial population. Considering initial population is the starting point of the Genetic Algorithm where the initial population comprises set of possible solutions to the specified problem.

\section{DESIGN PROBLEM}

\subsection{Design of FIR Filters}

Transfer function in z-domain of a linear, time-invariant digital filter is represented by the following equation: $H(z)=\frac{B(z)}{A(z)}=\frac{b_{0}+b_{1} z^{-1}+b_{2} z^{-2}+\cdots+b_{N} z^{-N}}{1+a_{1} z^{-1}+a_{2} z^{-2}+\cdots+a_{M} z^{-M}} \quad \ldots \ldots \ldots . . .(1) \quad$ This form basically represents a recursive filter with both the inputs (Numerator) and outputs (Denominator), that leads to an Infinite Impulse Response characteristics, when the 
denominator is made equal to unity then this leads to a Finite Impulse Response Filter [23]. FIR filters are extensively used in signal processing applications because it has the capability to realize a linear phase characteristic and to assure stability. Design of digital FIR filter involves calculation of filter transfer function coefficients $h_{n}, n=0,1, \ldots, N$ that provide target frequency response. For $\mathrm{N}$ as even and evenly symmetric impulse response, $h_{n}=h_{N-n}$, the magnitude response $H(\omega)$ can be represented by the following equation [24]:

$H(\omega)=\sum_{n=0}^{M} a_{n} \cos n \omega$, where $M=\frac{N}{2}$,

$a_{0}=$

$h_{M}, a_{n}=2 h_{M-n}, n=1,2, \ldots, M \ldots$ (2)

Impulse response of a linear time invariant FIR filters can be represented in sequence of filter coefficients considering the following equation:

$y_{n}=\sum_{k=0}^{N} h_{k} x_{n-k}$

\subsection{Design of FIR Filter using Window Function}

The elementary notion behind the use of Window functions to design filters is represented below $[25,26,27]$ :

$$
\begin{gathered}
H_{d}\left(e^{j \omega}\right)=1 \text { for } 0<\omega \leq \omega_{\text {cutoff }} \\
H_{d}\left(e^{j \omega}\right)=0 \text { for } \omega>\omega_{\text {cutoff }}
\end{gathered}
$$

This means ideal frequency response of the desired filter must be equals to 1 in the pass band, and equals to 0 in the stop band, the filter impulse response can be obtained by obtaining Discrete Fourier Transform (DFT) of the ideal frequency response. To design a Finite Impulse Response (FIR) filter, the time domain filter coefficients $\left(h_{d}(n)\right)$ can be restricted in number by multiplying by a window function of a finite width [18]. Most basic window function is rectangular window function [28]; a rectangular window of length $M$ can be expressed by the following equation:

$w_{R}(n)=1$ for $n=0,1,2, \ldots \ldots . M-1$

$=0$ elsewhere

The unit sample response $h(n)$ of the FIR filter is represented by the equation below:

$h(n)=\left(h_{d}(n)\right) w_{R}(n)$

Substituting $w_{R}(n)$ from eqn. $9, h(n)$ is limited to the length M,

$$
h(n)=h_{d}(n) \text { for } n=0,1,2, \ldots \ldots ., M-1
$$

$=0$ elsewhere
Frequency response of FIR filter is obtained by performing Fourier transform of eqn. 10, i.e.

$H(\omega)=F T\left\{h_{d}(n) \cdot w(n)\right\}$

As Fourier transform of the multiplication of two signals is equals to the convolved form desired frequency response and the window function, $H(\omega)$ can be stated as following:

$H(\omega)=H_{d}(\omega) \otimes W(\omega)$

Because of this convolution $H(\omega)$ have the smoothing effect and the side lobes of $W(\omega)$ produces the undesirably higher oscillation in $H(\omega)$. Therefore the width of the window is required to modify to reduce the effects of the side lobes and finally to make the filter frequency response more close to the ideal one. The window function must also be tapering down to zero at the ends increasing the width of the transition region between the pass and stop bands. At the same time this will lower the side lobe levels outside the pass band.

\section{KAISER WINDOW}

Kaiser Window permits separate control on the width of the main lobe and attenuation of the side lobes. Kaiser window is defined by the following equation:

$$
w_{k}(n)=\left\{\frac{I_{0}\left\{\left[1-\left(\frac{n-\alpha}{\alpha}\right)^{2}\right]^{\frac{1}{2}}\right\}}{I_{0}(\beta)} \text { for } 0 \leq n \leq N\right.
$$

$=0$ elsewhere

Here, $\alpha=\frac{N}{2}$, and $I_{0}$ is the first kind $0^{\text {th }}$ order modified Bessel function [28]. Here, $(\mathrm{N}+1)$ is the length of the window, $N=\frac{A-8}{2.285 \Delta \omega}$, If the transition width is $\Delta \omega \Delta \omega=\omega_{\text {stop_band }}-$

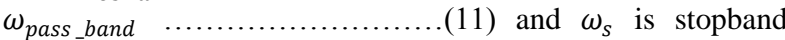
edge frequency and $\omega_{p}$ is passband edge frequency. $\beta$ is the shape of the window which can be selected independently. There is ripple of $\pm \delta_{1}$ in the passband and $\delta_{2}$ in the stopband. For the FIR filter design using Kaiser Window, minimum ripple of $\delta_{1}$ and $\delta_{2}$ is considered. Let the minimum ripple be represented by $\delta$. If attenuation is defined in $\mathrm{dB}, A=$ $-20 \log _{10} \delta$

Value of $\beta$ can be found out by using the following equations:

$$
\begin{aligned}
& \beta=0.1102(A-8.7) \text { for } A>50 \\
& \beta=0.5842(A-21)^{0.4}+0.07886(A-21)
\end{aligned}
$$

\begin{tabular}{|c|c|c|}
\hline Name of Window & Time-domain sequences, $\omega(n), 0 \leq n \leq M$ & Width of Main Lobe and \\
\hline Rectangular & 1 & $\begin{array}{l}\text { Narrowest main lobe about } \frac{4 \pi}{M+1} \& \text { large side lobes } \\
\text { about }-13 \mathrm{~dB} .\end{array}$ \\
\hline Barlett (Triangular) & $1-\frac{2\left|n-\frac{M-1}{2}\right|}{M-1}$ & $\begin{array}{c}\text { Medium main lob of } \frac{8 \pi}{M} \& \text { Side lobs of }-25 \mathrm{~dB} \text {. } \\
\text { Leakage Factor } 0.28 \% \text {. }\end{array}$ \\
\hline Blackman & $0.42-0.5 \cos \frac{2 \pi n}{M-1}+0.8 \cos \frac{4 \pi n}{M-1}$ & $\begin{array}{l}\text { Large main lob } \frac{12 \pi}{M} \& \text { Very good side lobs- } 57 \mathrm{~dB} . \\
\text { Leakage Factor } 0 \% \text {. }\end{array}$ \\
\hline Hamming & $0.54-0.46 \cos \frac{2 \pi n}{M-1}$ for $\mathrm{n}=0,1, \ldots, \mathrm{M}-1$ & $\begin{array}{c}\text { Medium main lob } \frac{8 \pi}{M} \& \text { Good side lobs }-41 \mathrm{~dB} \text {. } \\
\text { Leakage Factor } 0.03 \% \text {. }\end{array}$ \\
\hline
\end{tabular}

for $21 \leq A \leq 50$,

$\beta=0$ for $A<21$

Table 1. Comparison of Kaiser Window with other Windows 


\begin{tabular}{|c|c|c|c|}
\hline Hanning & $\frac{1}{2}\left(1-\cos \frac{2 \pi n}{M-1}\right)$ & For $n=0,1, \ldots, M-1$ & $\begin{array}{c}\text { Medium main lob } \frac{8 \pi}{M} \& \text { side lobes }-31 \mathrm{~dB} \text {. Leakage } \\
0.05 \% \text {. }\end{array}$ \\
\hline Tukey & $\begin{array}{l}\frac{1}{2}\left[1+\cos \left(\pi \frac{|n|-\alpha \frac{N}{2}}{(1-\alpha) \frac{N}{2}}\right)\right] \\
=0\end{array}$ & $\begin{array}{l}\text { when } \frac{\alpha N}{2} \leq|n| \leq \frac{N}{2} \\
\text { when } 0 \leq|n| \leq \frac{\alpha N}{2}\end{array}$ & $\begin{array}{l}\text { Main Lobe width }-3 \mathrm{~dB}, \text { relative sidelobes } \\
\text { attenuation }-15.1 \mathrm{~dB} \text {. Leakage Factor } 3.57 \% \text {. }\end{array}$ \\
\hline Kaiser & $\begin{array}{l}\frac{I_{0}\left\{\beta\left[1-\left(\frac{n-\alpha}{\alpha}\right)^{2}\right]^{\frac{1}{2}}\right\}}{I_{0}(\beta)} \\
=0 \quad \text { (elsewhere })\end{array}$ & (for $0 \leq n \leq M$ ) & $\begin{array}{l}\text { Kaiser Window function has an independent } \\
\text { parameter } \alpha \text {. By choosing the value of } \alpha \text { and the filter } \\
\text { length } N \text { arbitrary specifications can be achieved in } \\
\text { Lowpass (LP), highpass (HP), bandpass (BP) and } \\
\text { bandstop (BS) filters. By changing value of } \beta \text { and the } \\
\text { length of the filter main lobe width and side lobes } \\
\text { attenuation can be adjusted. }\end{array}$ \\
\hline
\end{tabular}

\section{GENETIC ALGORITHM BASED APPROACH}

Genetic Algorithms mimics the mechanism of natural evolutionary principles introduced by Charles Darwin [29]. This algorithm belongs to most challenging type of search algorithms known as Heuristic Search Algorithm. Heuristic Search Algorithms exploit additional knowledge about the problem that supports to search directly in more favorable paths [30]. They represent an intelligent manipulation of a random search used to solve optimization problems although randomized, GAs are by no means random, instead of, they exploit earlier information to drive the search to the region of better performance within the search space.

GA encodes all the data of a search space into a simple string called as a chromosome, which is usually of a fixed length. Each chromosome has a fitness value. GA is suitable for solving optimization problems. A typical GA can be described as follows [31]:

Step 1: GA starts with a set of possible solutions or chromosomes called initial population. Fitness value of each individual is computed.

Step 2: A set of chromosomes is then selected using a certain kind of selection procedure.

Step 3: These chromosomes are used to produce a new population using genetic operator crossover followed by another genetic operator mutation.

Step 4: Fitness value of each chromosome is computed.

Step 1 to Step 4 is repeated several times until the required criterion is satisfied. New population progresses by taking the solutions from the preceding populations.

The basic advantage of this algorithm is that it has the capability to handle a number of chromosomes at the same time, where each chromosome presents a different solution to a given problem. There are a remarkable number of fields where Genetic algorithms have applications, like digital signal processing, image processing, data clustering, path finding, project management, portfolio management, etc.

A generic selection procedure has been implemented as follows [32].

Step 1: The fitness function is calculated for each chromosome, providing fitness values which are then normalized.

Step 2: The population is arranged in ascending order according to the fitness values.
Step 3: Accumulated normalized fitness values are obtained (Accumulated fitness value of a chromosome $=$ Fitness value of that chromosome + the fitness values of all the previous chromosomes). The accumulated fitness of the last individual must be 1 .

Step 4: A random value should preferably be chosen between 0 and 1.

Step 5: The selected chromosome will be the first one whose accumulated normalized value is greater than the randomly chosen value.

Step 1 to step 5 are repeated until the initial population converges.

This selection method is accustomed with fitness proportionate selection or Roulette-wheel selection [33] and was proposed by Holland. Probability of each individual in this selection procedure can be described by the following equation [34]:

$P[$ Individual $i$ is chosen $]=\frac{F_{i}}{\sum_{j=1}^{P_{0} p \text { size }} F_{j}} \ldots \ldots \ldots \ldots \ldots$ (14)

$F_{i}$ stands for fitness value of the $i^{\text {th }}$ individual in the population. $F_{j}$ represents fitness value of the $\mathrm{j}^{\text {th }}$ individual in the population.

\section{PROPOSED ALGORITHM}

Step 1: Filters are implemented using Kaiser Window function with different values of passband and stopband ripples (passband ripple varies from 0.01 to 0.40 and stopband ripple varies from 0.09 to 0.49 ). Corrupted signal is then filtered using the implemented filters. Filtered signals are kept in a matrix. This matrix is termed as initial population. Each row of the matrix containing a filtered signal termed as chromosome.

Step 2: For determination of fitness values of chromosomes, following equation is followed.

$\left(\beta=\frac{(\text { Corrupted Signal }- \text { Filtered Signal })}{\text { Filtered Signal }}\right.$

Finally the value of $10 \log _{10}(\beta)$ has been used as the fitness value.

Step 3: Based on fitness values a set of filtered signals has been selected from the initial population using Roulette Wheel Selection procedure.

Step 4: Single point Crossover is performed with $100 \%$ probability in between the selected set of chromosomes and off springs are generated. 
Step 5 Mutation is performed on the offspring chromosomes with $25 \%$ probability.

Step 6: Replacement of parent signals by off-spring signals with better fitness values than the parent signals.

Step 7: Signal with highest fitness value has been obtained as best offspring signal.

Step 8: Repeat Step 4 to Step 7 N (N=10) times.

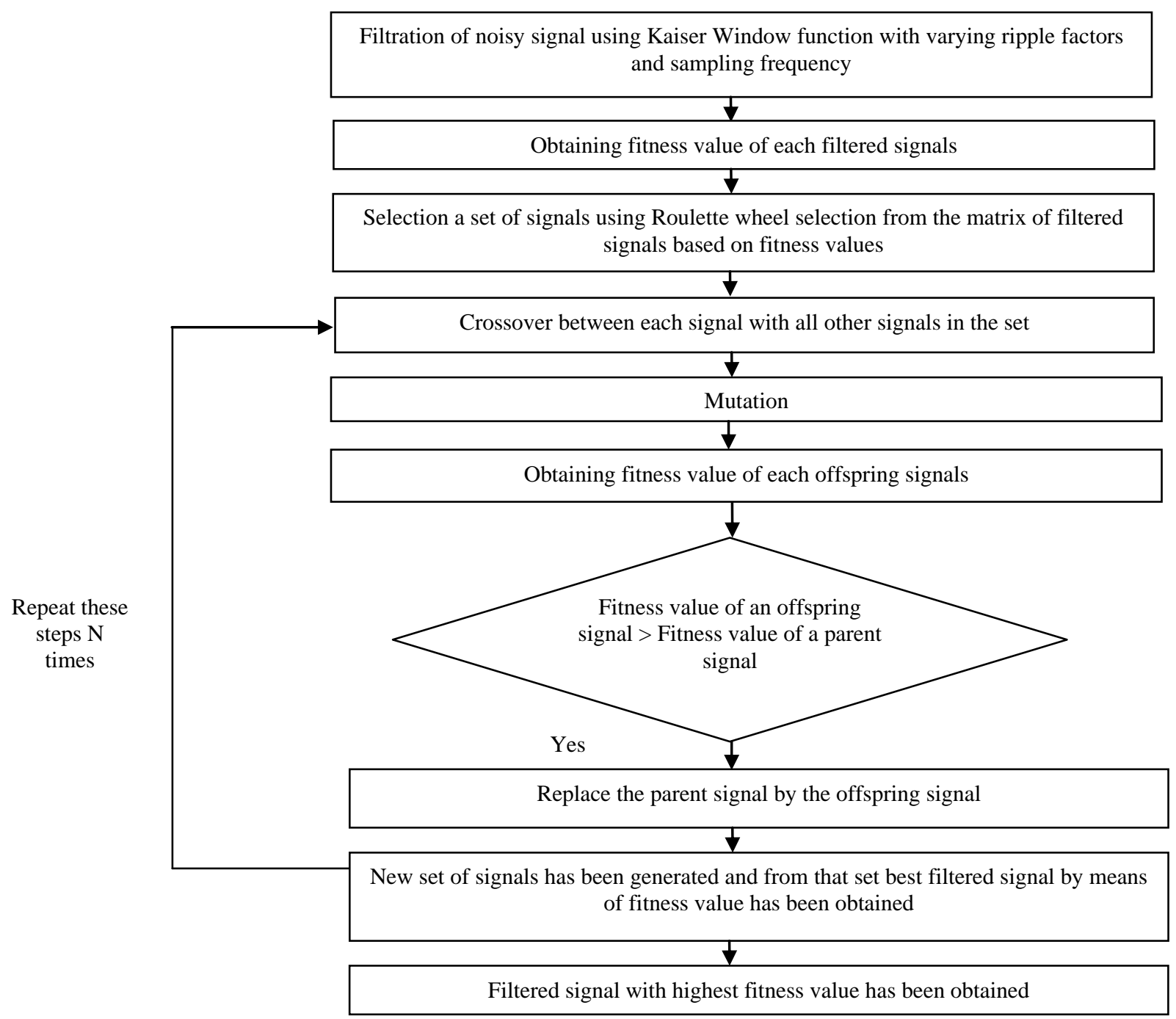

Fig 1: Flowchart of Proposed Algorithm

\section{RESULTS AND DISCUSSION}

Proposed algorithm is capable of identifying little amount of noise present in the signal and excluding it from the signal. Hence, it is very much useful for de-noising biomedical signals, where very little amount of noise may cause erroneous diagnosis.

\subsection{Case Study}

A heart Sound Signal without any noise has been collected from a diagnostic center. Random noise has been incorporated in the original heart sound signal. Original Heart Sound Signal and Noisy Heart Sound Signal have been shown in Fig. 2 (a) \& (b). SNR (Signal to Noise Ratio) of the corrupted signal is 2.9126 and correlation of the corrupted signal is 0.8015 .

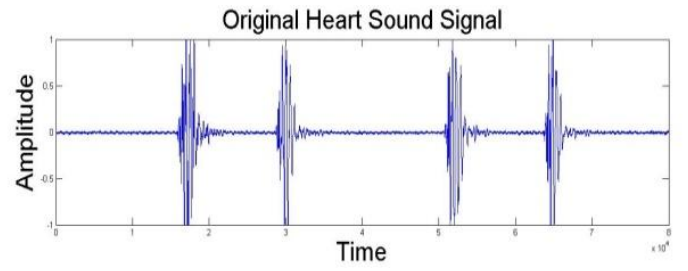

Figure 2(a): Original Heart Sound Signal 


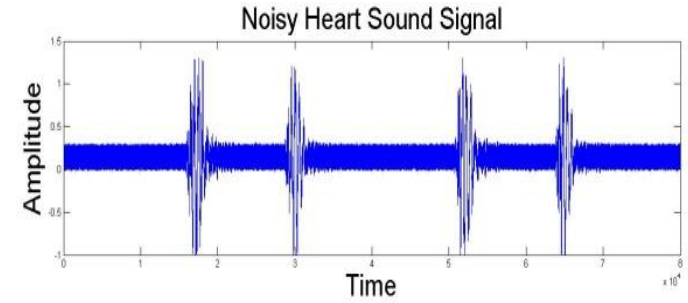

Figure 2(a): Noisy Heart Sound Signal

Proposed Algorithm has been performed over the corrupted heart sound signal for a range of sampling frequency (500012000) Hz. Filtered heart sound signals obtained by the proposed algorithm for different sampling frequencies are then compared with the original signal. SNR and Correlation values of the filtered signals are shown in Table 2. From Table 2 it can be seen that the filtered signal obtained by the algorithm at sampling frequency of $7000 \mathrm{~Hz}$ has the highest SNR (Signal to Noise Ratio) value and the filtered signal obtained by the algorithm at sampling frequency of $8000 \mathrm{~Hz}$ has the highest Correlation value.

Table 2. Variation of SNR and Correlation values of Filtered Heart Sound signals withSampling Frequency

\begin{tabular}{|c|c|c|}
\hline $\begin{array}{c}\text { Sampling } \\
\text { frequency }\end{array}$ & SNR & Correlation \\
\hline 5000 & 10.4374 & 0.9327 \\
\hline 6000 & 11.0880 & 0.9566 \\
\hline $\mathbf{7 0 0 0}$ & $\mathbf{1 2 . 0 0 1 8}$ & $\mathbf{0 . 9 7 3 4}$ \\
\hline $\mathbf{8 0 0 0}$ & $\mathbf{1 1 . 3 3 8 3}$ & $\mathbf{0 . 9 9 7 9}$ \\
\hline 9000 & 8.0536 & 0.9975 \\
\hline 10000 & 5.5888 & 0.9970 \\
\hline 11000 & 3.6374 & 0.9965 \\
\hline 12000 & 2.0305 & 0.9959 \\
\hline
\end{tabular}

Variations of SNR and Correlation value of the filtered signals for different sampling frequencies are shown in Fig. 3 (a) \& (b).

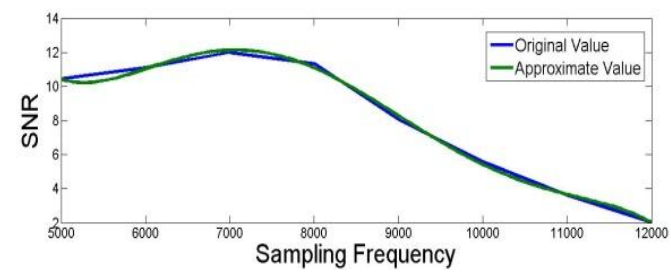

Figure 3(a): Plot of SNR-Sampling Frequency

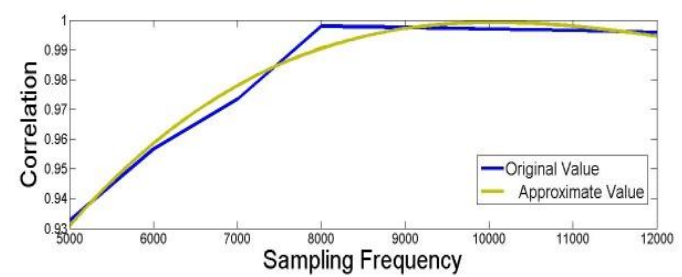

Figure 3(b): Plot of Correlation value-Sampling Frequency

Filtered heart sound signals obtained by the proposed algorithm with sampling frequency $7000 \mathrm{~Hz}$ and $8000 \mathrm{~Hz}$ have been shown in Fig. 4 (a) \& (b).

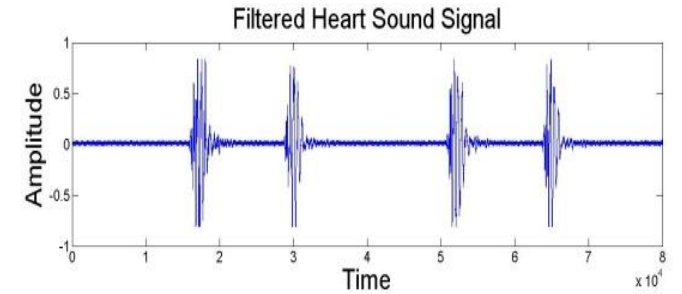

Fig 4(a): Filtered (Best offspring) Signal at Sampling Frequency $7000 \mathrm{~Hz}$

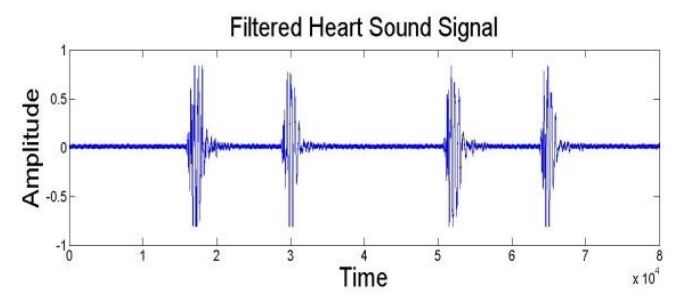

Fig 4(b): Filtered (Best offspring) Signal at Sampling Frequency $8000 \mathrm{~Hz}$

\section{CONCLUSIONS}

In this correspondence, we have proposed a new algorithm of de-noising method with GA based response. Experimental results and corresponding pictorial representations render that the whole adaptive algorithm has been successively used to produces the best response at the range of $7-8 \mathrm{KHz}$ sampling frequency which varies from 5 to $12 \mathrm{KHz}$ and simultaneously ensure the successive application of the proposed method enhances the optimization efficacy to reduce noise spectrum from the original signals-noise spectra. Signal with least noise at $7 \mathrm{KHz}$ sampling frequency poses SNR of 12.0018. Signal with highest correlation with the original signals at $8 \mathrm{KHz}$ sampling frequency poses Correlation value of 0.9979 . Proposed algorithm is capable of identifying little amount of noise present in the signal and excluding it from the signal

\section{ACKNOWLEDGMENTS}

This work is partially supported by UGC-BSR Research Start-up Grant under Faculty Research Promotion Scheme, University Grants Commission, Government of India.

\section{REFERENCES}

[1] Chaplot, R., Paliwal, A. 2013. Low Power Reconfigurable FIR Filter Based on Window Techniques for On Chip Network, In Proceedings of the International Conference on Signal Processing and Communication (ICSC).

[2] Jeong, W., Meimand, H. M., Park, J. et.al. "Computation Sharing Programmable FIR Filter for Low-Power and High-Performance Applications", IEEE Journal of SolidState Circuits, 2004.

[3] Roh, T., Dumitrescu, B. "Multidimensional FIR Filter Design Via Trigonometric Sum-of-Squares Optimization", IEEE Journal Of Selected Topics In Signal Processing, 2007.

[4] Ye, W.B., Yu, Y.J. "Single-Stage and Cascade Design of High Order Multiplierless Linear Phase FIR Filters Using Genetic Algorithm", IEEE Transactions on Circuits and Systems-I: Regular Papers, 2013.

[5] Kundu, D., Suresh, K., Das, S., ghosh, S. et al. 2009. Design of optimal digital IIR filters by using a Bandwidth Adaptive Hermony Search Algorithm, In 
Proceedings of World Congress on Nature \& Biologically Inspired Computing.

[6] PrasadBattula, V.V.K.D.V., Vasantha, V., Sravanthi, V. et. al. "Design of FIR Filter by using Sharing Multiplier with Low Delay", International Refereed Journal of Engineering and Science (IRJES), 2012.

[7] Awad, A.M. 2010. Adaptive Window Method for FIR Filter Design, In Proceedings of IEEE Conference on Open Systems (ICOS).

[8] Shayesteh, M.G., Mottaghi-Kashtiban. M. 2009. FIR filter design using a new window function, In Proceedings of $16^{\text {th }}$ International Conference on Digital Signal Processing.

[9] Karmakar, S., Ghosh, K., Sen, S., Sarkar, S.et.al. 2008. An alternative Gaussian Window approach for FIR filter Design, In Proceedings of International Conference on Signal Processing, Communication and Networking.

[10] Eleti, A.A.. 2013. FIR Digital Filter Design by using Windows method with Matlab, In Proceedings $14^{\text {th }}$ International Conference on Sciences and Techniques of Automatic Control and Computer Engineering (STA).

[11] Elektron, E., Bolumu, M., Avci, K. et.al. 2013. Performance analysis of Kaiser-Hamming Window for nonrecursive digital filter design, In Proceedings of $21^{\text {st }}$ Conference on Signal Processing and Communications Applications.

[12] J. Barros, "On the Use of the Hanning window for harmonic analysis in the standard framework", IEEE Transactions on Power Delivery, 2006.

[13] Avci, K., Nacaroglu, A. "Design of Non-recursive Digital Filters Using the Exponential Window", International Journal of Advances in Electrical and Electronics Engineering, volume: 2 (2), 308-316.

[14] Avalos Ochoa, J.G., Escamilla Hernández, E., Sánchez García, J.C., MartínezCarrada, H.M.et.al. 2014. FIR Filters Using Atomic Window Functions Implemented on the DSP C6713, In Proceedings of International Conference on Electronics, Communications and Computers.

[15] Avci, K., Nacaroğlu, A. 2008. High quality low order non recursive digital filters design using modified Kaiser Window, In Proceedings of $6^{\text {th }}$ International Symposium on Communication Systems, Networks and Digital Signal Processing.

[16] Roy, T.K., Morshed, M. 2013. Performance Analysis of Low Pass FIR Filters Design Using Kaiser, Gaussian and Tukey Window Function Methods, In Proceedings of 2013 2nd International Conference on Advances in Electrical Engineering (ICAEE 2013).

[17] Lin, Y.P. 1998. A Kaiser Window approach for the Design of Prototype Filters of Cosine modulated Filter banks, IEEE Signal Processing Letters, (1998), 132-134.

[18] Kaur, T. 2014. "Approach for design of FIR filter using Kaiser Window", ISTP Journal of Research in Electrical and Electronics Engineering (ISTP-JREEE), Proceedings of 1st International Conference on Research in Science, Engineering \& Management.

[19] Ye, W.B., Yu, Y.J., H. Zhao.et.al. 2013. Sparse FIR Filter Design Based on Genetic Algorithm, In
Proceedings of International Symposium on Circuits and Systems.

[20] Chauhan, R.S., Arya, S. K., An Optimal Design of FIR Digital Filter Using Genetic Algorithm, Communications in Computer and Information Science, 51-56.

[21] Kaur, R., Pattreh, M.S., Dhillon, M.S., "Digital IIR Filter Design using Real Coded Genetic Algorithm", International Journal of Information Technology and Computer Science, June 2013.

[22] Ababneh, J. I., Bataineh, M.H., Linear phase FIR filter design using particle swarm optimization and genetic algorithms, ELSEVIER Digital Signal Processing, July 2008, 657-668.

[23] https://en.wikipedia.org/wiki/Digital_filter

[24] T. Sasahara, K. Suyama. 2015. An ACO Approach for Design of CSD Coefficient FIR Filters, In Proceedings of APSIPA Annual Summit and Conference.

[25] T. Singh, H.S. Joshan, "Design of Low Pass Digital FIR Filter Using Cuckoo Search Algorithm", International Journal of Engineering Research and Applications, August 2014.

[26] A. Mallick, S. Dutta, S. Roy, U. Kumar, A Chattopadhyay, D. Chakraborty, S. Nag. 2014. Cuckoo Search Optimization based design of linear phase FIR filters: A comparison approach, In Proceedings of International Conference on Green Computing Communication and Electrical Engineering (ICGCCEE).

[27] Neha, Ajay Pal Singh, "Design of Linear Phase Low Pass FIR Filter using Particle Swarm Optimization Algorithm”, International Journal of Computer Applications, July 2014.

[28] Sharma, S. 2009. Book on Digital Signal Processing (with Matlab Programs.

[29] Kumar, M., Rawat, T.K., Upadhyay, K. et.al. 2014. Optimal Design of Weighted Least Square Based Fractional Delay FIR Filter Using Genetic Algorithm, (2014), 53-58.

[30] http://www.cs.utexas.edu/ mooney/cs343/slidehandouts/heuristic-search.4.pdf

[31] Najjarzadeh, M., Ayatollahi, A. 2013. A Comparison between Genetic Algorithm and PSO for Linear Phase FIR Digital Filter Design, In Proceedings of $9^{\text {th }}$ International Conference on Signal Processing.

[32] Jaiswal, H.L., Singh, I., Chandra sekaran, K., Agrawal, D.et.al. An Evolutionary Approach to Optimizing Cloud Services, Computer Engineering and Intelligent Systems.

[33] Razali, N.M., Geraghty, J. 2011. Genetic Algorithm Performance with Different Selection Strategies in Solving TSP, In Proceedings of the World Congress on Engineering.

[34] Atanassov, K., Shannon, A., Pencheva, T. Modelling of a Roulette Wheel Selection Operator in Genetic Algorithms Using Generalized Nets, BIO Animation, 2009, 257-264 